\title{
Baseline Mac-2 Binding Protein Glycosylation Isomer Level Stratifies Risks of Hepatocellular Carcinoma in Chronic Hepatitis B Patients with Oral Antiviral Therapy
}

\author{
Tai-Chung Tseng a, b Cheng-Yuan Peng ${ }^{\mathrm{f}} \mathrm{g}$ Yao-Chun Hsuk \\ Tung-Hung Su ${ }^{a, b}$ Chia-Chi Wang ${ }^{\text {h, } i}$ Chun-Jen Liu ${ }^{a-c}$ \\ Hung-Chih Yang a, e Wan-Ting Yang ${ }^{b}$ Chia-Hsin Ling ${ }^{g}$ Ming-Lung Yu' Hsueh- \\ Chou Lai $^{9}$ Yasuhito Tanakam Mindie H. Nguyen ${ }^{\mathrm{n}}$ \\ Chen-Hua Liu ${ }^{a, b}$ Pei-Jer Chen ${ }^{a-c}$ Ding-Shinn Chen ${ }^{a-c, j}$ Jia-Horng Kao ${ }^{a-d}$ \\ a Division of Gastroenterology and Hepatology, Department of Internal Medicine, National Taiwan \\ University Hospital, Taipei, Taiwan; ${ }^{b}$ Hepatitis Research Center, National Taiwan University Hospital, \\ Taipei, Taiwan; ' Graduate Institute of Clinical Medicine, National Taiwan University College of \\ Medicine, Taipei, Taiwan; ${ }^{d}$ Department of Medical Research, National Taiwan University Hospital, \\ Taipei, Taiwan; ${ }^{e}$ Department of Microbiology, National Taiwan University College of Medicine, \\ Taipei, Taiwan; ${ }^{f}$ School of Medicine, China Medical University, Taichung, Taiwan; 9 Division of \\ Hepatogastroenterology, Department of Internal Medicine, China Medical University Hospital, \\ Taichung, Taiwan; h Division of Gastroenterology, Department of Internal Medicine, Taipei Tzuchi \\ Hospital, The Buddhist Tzuchi Medical Foundation, Taipei, Taiwan; 'School of Medicine, Tzu Chi \\ University, Hualien, Taiwan; ${ }^{j}$ Genomics Research Center Academia Sinica, Taipei, Taiwan; ${ }^{k}$ Division of \\ Gastroenterology and Hepatology, Department of Internal Medicine, E-Da Hospital/I-Shou University, \\ Kaohsiung, Taiwan; 'Hepatobiliary Division, Department of Internal Medicine, Kaohsiung Medical \\ University Hospital, Kaohsiung Medical University, Kaohsiung, Taiwan; ${ }^{m}$ Department of Virology and \\ Liver Unit, Nagoya City University Graduate School of Medical Sciences, Nagoya, Japan; ${ }^{\text {nDivision of }}$ \\ Gastroenterology and Hepatology, Stanford University Medical Center, Palo Alto, CA, USA
}

\section{Keywords}

Hepatitis B virus - Mac-2 binding protein glycosylation isomer - Hepatocellular carcinoma .

Fibrosis · Nucleos(t)ide analogue $\cdot$ Chronic hepatitis B

\begin{abstract}
Background and Aims: Mac-2 binding protein glycosylation isomer (M2BPGi) is a novel biomarker correlating with liver fibrosis stages. However, little is known about how it predicts risks of hepatocellular carcinoma ( $\mathrm{HCC}$ ) in chronic hepatitis $B(\mathrm{CHB})$ patients receiving longterm antiviral treatment. Materials and Methods: The study contained 2 parts. The first part

T.-C. Tseng and C.-Y. Peng shared the first authorship.


was to explore whether M2BPGi could be an HCC predictor in $899 \mathrm{CHB}$ patients receiving long-term entecavir therapy. The second part was to validate the findings in an independent cohort of 384 on-treatment CHB patients with more severe liver disease. Results: In the discovery cohort, there were 64 patients developing HCC within an average follow-up of 7.01 years. Our data showed that M2BPGi level was positively associated with HCC development. When stratifying the patients by an M2BPGi level of 1.73 (the third quartile), the high M2BPGi group was shown to have an increased HCC risk compared to the low M2BPGi group with hazard ratio of 5.80 (95\% $\mathrm{Cl} 3.50-9.60)$. Furthermore, we found that the M2BPGi level complements PAGE-B score, a well-validated HCC prediction model, to predict HCC development. Lastly, the cutoff was validated in the independent cohort, especially those with an intermediate PAGE-B score. Conclusions: In CHB patients receiving long-term antiviral treatment, serum M2BPGi level not only serves as an independent HCC predictor but also complements PAGE-B in stratifying HCC risks.

(c) 2020 The Author(s)

Published by S. Karger AG, Base

\section{Introduction}

Hepatitis B virus (HBV) infection is a global health problem and patients with chronic hepatitis $\mathrm{B}(\mathrm{CHB})$ are at risk of developing cirrhosis, hepatic decompensation, and hepatocellular carcinoma (HCC), resulting in over 1 million deaths peryear [1]. Fortunately prolonged nucleos(t) ide analogue (NA) treatment has been shown to reduce these liver-related complications [2, 3].

Although cirrhosis-related complications are minimized by pre-emptive antiviral therapy, the residual HCC incidence remains an important issue [4]. Since it is labor-intensive to have regular HCC surveillance in all CHB patients receiving anti-viral treatment, the unmet need is to introduce a novel biomarker to effectively stratify the on-treatment HCC risks, which may guide practicing physicians to provide individualized management [4].

Most models predicting HCC development in CHB patients with prolonged NA treatment adopt traditional predictors including host factors, liver function, and liver fibrosis severity [513]. However, only PAGE-B score has been well validated $[7,11,14]$. One of the reasons for the gap is the patients' cirrhosis status, which is an important variable of several prediction models, is usually determined by abdominal ultrasonography $[9,10]$, or transient elastography $[8,12]$. The former tool is limited by its sensitivity and accuracy due to the inter-observer variation, while the later tool is unlikely to be applied in primary care units [15]. A serum biomarker for evaluating liver fibrosis is expected to serve as an ideal tool to avoid both limitations [15]. Mac 2-binding protein glycosylation isomer (M2BPGi), which is a liver-specific glycosylation isomer of M2BP, has been shown to serve as a liver fibrosis marker in patients with different liver diseases [16]. Recent longitudinal data in chronic hepatitis $C$ patients suggested elevated serum M2BPGi level was associated with an increased HCC risk [17]. Although previous data suggested that M2BPGi could be an HCC predictor in CHB patients receiving long-term NA treatment [1823], none of the M2BPGi cutoffs has been validated yet. Furthermore, little is known about whether it complements current prediction models in predicting HCC development.

To address these critical issues, we conducted the current study, which consisted of 2 parts. In the discovery cohort study, we explored whether the serum M2BPGi level was associated with HCC development in CHB patients receiving long-term NA treatment and whether it served as a complementary HCC predictor on top of the PAGE-B prediction model, which is a well-validated model containing platelet count, age, and gender as predictive variables without taking into account liver cirrhosis status determined by ultrasonography or transient elastography [7]. In the validation cohort, we validated our finding in an independent cohort of CHB patients who were treated and followed up in different hospitals. 


\section{Liver Cancer}

\begin{tabular}{|c|c|}
\hline \multicolumn{2}{|c|}{ Liver Cancer 2020;9:207-220 } \\
\hline DOI: $10.1159 / 000504650$ & $\begin{array}{l}\text { (c) } 2020 \text { The Author(s). Published by S. Karger AG, Basel } \\
\text { www.karger.com/lic }\end{array}$ \\
\hline
\end{tabular}

Tseng et al.: M2BPGi and HBV-HCC Risks

\section{Materials and Methods}

Patient Cohorts

In the first discovery cohort study, online supplementary Figure 1 (for all online suppl. material, see www.karger.com/doi/10.1159/000504650) shows the inclusion and exclusion criteria of patients in this retrospective cohort. A total of 1,806 hepatitis B surface antigen (HBsAg)-positive patients aged above 28 years were consecutively enrolled between 2007 and 2016. All of the patients were either from the National Taiwan University Hospital, the China Medical University Hospital, or the Taipei Tzuchi Hospital [5, 6]. All of them were treatment-naïve and decided to receive long-term entecavir treatment, either due to HBV DNA $>2,000 \mathrm{IU} / \mathrm{mL}$, liver cirrhosis or suffering from liver decompensation caused by HBV reactivation [24, 25]. After excluding the patients without adequate serum samples, those with evidence of autoimmune hepatitis, alcoholic liver disease, or HCV coinfection, and those who received immunosuppressive drugs, 1,299 patients remained. We then excluded patients having HCC diagnosed either at baseline or within the first year of follow-up and those who were lost to follow-up within the first year. Finally, a total of 899 patients were included for subsequent analyses. During the treatment period, entecavir was shifted to tenofovir at the physician's discretion when there existed suboptimal viral suppression.

Findings from the aforementioned discovery cohort were extrapolated to an independent cohort for external validation, which consisted of 384 CHB patients enrolled from the E-Da Hospital and Kaohsiung Medical University Hospital (both in Kaohsiung, Taiwan). The details of how patients were enrolled and their baseline characteristics, the pattern of M2BPGi decline on NA monotherapy and the association with HCC incidences had been reported [18].

All the enrolled patients in both cohorts gave written informed consent as approved by the research Ethical Committees of each hospital.

\section{Data Collection}

Patients were tested for serological markers, including HBsAg, hepatitis B e antigen (HBeAg), anti-HBe, anti-HCV, and had liver function tests at baseline. Throughout the follow-up period, serum liver function tests, HBV DNA levels, and alpha-fetoprotein levels were assayed every 3-6 months and abdominal ultrasonography was performed every 6 months for HCC surveillance. Serum samples at baseline and follow-up were stored at $-20^{\circ} \mathrm{C}$ until analysis.

Diagnosis of Cirrhosis and HCC

Cirrhosis diagnosis is based on either histology, liver stiffness measurement $>13 \mathrm{kPa}$ (available after 2009), or ultrasonographic findings plus clinical features of thrombocytopenia, gastroesophageal varices, or ascites [26,27]. For the diagnosis of cirrhosis made via abdominal ultrasound, the findings had to be consistent on at least 2 occasions 6 months apart. HCC was diagnosed either by histology/cytology or by typical imaging findings (arterial enhancement and venous wash-out by contrast-enhanced computerized tomography or magnetic resonance imaging scanning) in hepatic nodules larger than $1 \mathrm{~cm} \mathrm{[28].}$

Serological Assays

Serum HBsAg, HBeAg, anti-HBe, and anti-HCV were tested using commercial assays (Abbott Laboratories, Abbott Park, IL, USA).

Quantification of Serum HBV DNA

Serum HBV DNA was quantified using the COBAS AmpliPrep-COBAS TaqMan HBV test with the lower limit of detection of $20 \mathrm{IU} / \mathrm{mL}$.

\section{Measurement of Serum M2BPGi}

M2BPGi level was directly measured with the HISCL M2BPGi regent (Sysmex Co., Kobe, Japan) using a fully automated immunoanalyzer, HISCL-800 (Sysmex Co., Kobe, Japan).

\section{Statistical Analysis}

HCC was defined as the endpoint. The clinical follow-up started at the time of starting antiviral treatment. The person-years were censored on the date of HCC development, death, the last date of follow-up, or May 31, 2018, whichever came first. The cumulative incidence of HCC stratified by different variables was derived using the Kaplan-Meier curve analysis and the log rank test was used to test for the statistical difference. 
Tseng et al.: M2BPGi and HBV-HCC Risks

Mean and SD were calculated for continuous variables and percentages were used for categorical variables. Cox proportional hazards regression model was adopted to calculate the crude and multivariateadjusted hazard ratios (HR) of HCC. Statistical significance of all tests in the longitudinal cohort studies was defined as $p<0.05$ by two-tailed tests. All analyses were performed using Stata statistical software (version 10.0; Stata Corp, College Station, TX, USA).

\section{Results}

Discovery Cohort Study: Baseline Characteristics and Follow-Up Results of 899 Patients with Long-Term Entecavir Treatment

Table 1 shows the baseline characteristics of the 899 patients when they started longterm entecavir treatment. Of them, 669 (74.7\%) were males; $773(86.0 \%)$ had ALT levels $\geq 40$ $\mathrm{U} / \mathrm{L} ; 173(19.2 \%)$ had cirrhosis diagnosed at baseline. In terms of viral factors, 354 (39.4\%) had positive HBeAg; 839 (93.3\%) had HBV DNA levels $\geq 2,000 \mathrm{IU} / \mathrm{mL}$.

\section{A Positive Relationship between Baseline M2BPGi Level and HCC Risk}

There were 6,303.16 person-years of follow-up, with a mean follow-up period of $7.01 \pm$ 3.36 years. Throughout the follow-up period, 64 patients developed HCC with an incidence rate of 1.02 cases per 100 person-years.

We first correlated baseline serum M2BPGi levels with HCC incidence, and found that serum M2BPGi levels were positively associated with HCC incidence rates ( $p$ for trend $<0.001$ ). To identify a useful cutoff, all the patients were divided into 4 groups according to baseline M2BPGi quartiles: group I M2BPGi <0.48; group II $0.48 \leq \mathrm{M} 2 \mathrm{BPGi}<0.85$; group III $0.85 \leq \mathrm{M} 2 \mathrm{BPGi}<1.73$ and group IV M2BPGi $\geq 1.73$. The annual incidence rates of $\mathrm{HCC}$ increased from $0.16 \%$ (95 CI $0.05-0.51 \%$ ) in group I to $2.92 \%$ (95\% CI $2.13-3.99 \%$ ) in group IV. When compared to group I, there was a moderate increase in HCC risk in group III $(p=0.006)$, and a marked increase in group IV ( $p<0.001$; Fig. 1a). After adjustment for cirrhosis status, only group IV remained as an independent risk factor.

\section{Comparing the Predicting Performance of HBV-Related HCC among Different M2BPGi \\ Cutoffs Reported in the Literature}

To validate whether 1.73 is an optimal cutoff, we compared its prediction performance using Youden index with other M2BPGi cutoffs once reported for predicting HBV-related HCC development or recurrence either in on-treatment patients [19-23], treatment-naïve patients $[20,29-32]$, or patients receiving surgical resection [19,33]. Among all the 12 cutoffs, M2BPGi of 1.73 was shown to be a better cutoff due to the highest index (online suppl. Table 1). Considering M2BPGi of 1.79 has been proposed as the cutoff to define CHB patients with liver fibrosis of F3 or more [34], which is close to 1.73, we decided to choose the third quartile level of 1.73 as our cutoff in this study.

\section{HCC Risks after Dividing the Patients using M2BPGi Cutoff of 1.73}

After categorizing the patients with the M2BPGi level of 1.73, the high M2BPGi group had an increased HCC risk with the HR of 5.80 (95\% CI 3.50-9.60) when compared to the low M2BPGi group (Fig. 1b, Table 2). Among other factors, older age, male sex, lower platelet count, and presence of cirrhosis were risk factors, and cirrhosis itself was the strongest risk factor of HCC, with the HR of 11.00 (95\% CI 6.50-18.59; Table 2). Further multivariable analysis showed that $\mathrm{M} 2 \mathrm{BPGi} \geq 1.73$ remained as an independent risk factor with a HR of 2.12 (95\% CI 1.17-3.86) in addition to increased age, male sex, platelet count, and cirrhosis at baseline (Table 2). 


\section{Liver Cancer}

\begin{tabular}{|c|c|}
\hline \multicolumn{2}{|l|}{ Liver Cancer 2020;9:207-220 } \\
\hline DOI: 10.1159/000504650 & $\begin{array}{l}\text { (c) } 2020 \text { The Author(s). Published by S. Karger AG, Basel } \\
\text { www.karger.com/lic }\end{array}$ \\
\hline
\end{tabular}

Tseng et al.: M2BPGi and HBV-HCC Risks

Table 1. Comparison of baseline characteristics between discovery and validation cohorts of patients with chronic HBV infection receiving long-term antiviral treatment

\begin{tabular}{|c|c|c|c|}
\hline & $\begin{array}{l}\text { Discovery cohort } \\
(n=899)\end{array}$ & $\begin{array}{l}\text { Validation } \\
\text { cohort }(n=384)^{\S}\end{array}$ & $p$ value $\mathrm{e}^{\#}$ \\
\hline Gender & & & 0.73 \\
\hline Female & $230(25.58)$ & $102(26.56)$ & \\
\hline Male & $669(74.42)$ & $282(73.44)$ & \\
\hline Age at enrolment, years & $48(16)$ & $48(19)$ & 0.03 \\
\hline Platelet count, $10^{3} / \mu \mathrm{L}$ & $173(74)$ & $155(103)$ & $<0.001$ \\
\hline Serum ALT levels, U/L & & & 0.15 \\
\hline$<40$ & $126(14.02)$ & $42(10.94)$ & \\
\hline$\geq 40$ & $773(85.98)$ & $342(89.06)$ & \\
\hline HBeAg & & & 0.62 \\
\hline Negative & $542(60.49)$ & $226(58.85)$ & \\
\hline Positive & $354(39.51)$ & $158(41.15)$ & \\
\hline Serum HBV DNA, $\log _{10} \mathrm{IU} / \mathrm{mL}$ & $6.13(2.63)$ & $6.35(2.58)$ & 0.20 \\
\hline Baseline cirrhosis & & & $<0.001$ \\
\hline No & $726(80.76)$ & $189(49.22)$ & \\
\hline Yes & $173(19.24)$ & $195(50.78)$ & \\
\hline M2BPGi, COI & $0.85(1.25)$ & $1.68(3.62)$ & $<0.001$ \\
\hline M2BPGi, COI (quartiles) & & & $<0.001$ \\
\hline M2BPGi <0.48 (group I) & $227(25.25)$ & $40(10.42)$ & \\
\hline $0.48 \leq$ M2BPGi <0.85 (group II) & $221(24.58)$ & $61(15.89)$ & \\
\hline $0.85 \leq \mathrm{M} 2 \mathrm{BPGi}<1.73$ (group III) & $222(24.69)$ & $94(24.28)$ & \\
\hline M2BPGi $\geq 1.73$ (group IV) & $229(25.47)$ & $189(49.22)$ & \\
\hline PAGE-B score* & & & $<0.001$ \\
\hline Low $($ score $<10)$ & $169(18.80)$ & 69 (17.97) & \\
\hline Intermediate (score 10-17) & $485(53.95)$ & $169(44.01)$ & \\
\hline High $($ score $>17$ ) & $245(27.25)$ & $146(38.02)$ & \\
\hline \multicolumn{4}{|l|}{ METAVIR fibrosis stage $^{\dagger}$} \\
\hline $\mathrm{F} 0 / 1$ & $64(23.46)$ & Not available & \\
\hline $\mathrm{F} 2$ & $78(28.47)$ & & \\
\hline F3 & $66(24.09)$ & & \\
\hline F4 & $66(24.09)$ & & \\
\hline
\end{tabular}

All the continuous factors are expressed as median value (interquartile range, IQR) and all the categorical factors are expressed as $n(\%) .{ }^{\S}$ There were 245 patients $(63.80 \%)$ receiving entecavir or tenofovir while other received lamivudine, adefovir or telbivudine. ${ }^{*} p$ values calculated by Fisher's exact test for categorical variables and Mann-Whitney U test for continuous variables. * The PAGE-B score is calculated as age $(<30$ years $=0 ; 30-39=2 ; 40-49=4 ; 50-59=6 ; 60-69=8 ; \geq 70=10)+$ sex $($ male $=6$; female $=0)+$ platelet count $\left(\times 1,000 / \mathrm{mm}^{3} ; \geq 200=0 ; 100-199=6 ;<100=9\right) .{ }^{\dagger} 274$ patients receiving liver biopsy before initiating entecavir treatment. ALT, alanine transaminase; HBeAg, hepatitis B e antigen; HBV, hepatitis B virus; M2BPGi, Mac-2 binding protein Glycosylation isomer; COI, cut-off index.

\section{M2BPGi Kinetics and HCC Risks}

To validate these findings, we then explored the temporal relationship between M2BPGi levels and HCC development during the follow-up period. The M2BPGi kinetics was explored in 162 patients with available serum samples during follow-up, including 36 HCC patients and 126 patients without HCC development. The serum M2BPGi levels were determined at 3 different time points: baseline, achieving undetectable viral load, and the end of follow-up or when HCC diagnosed. The median levels of M2BPGi in HCC and non-HCC patients are shown in Figure 1c. The M2BPGi levels of the HCC group were consistently higher than those of the non-HCC group at all 3 time points. 


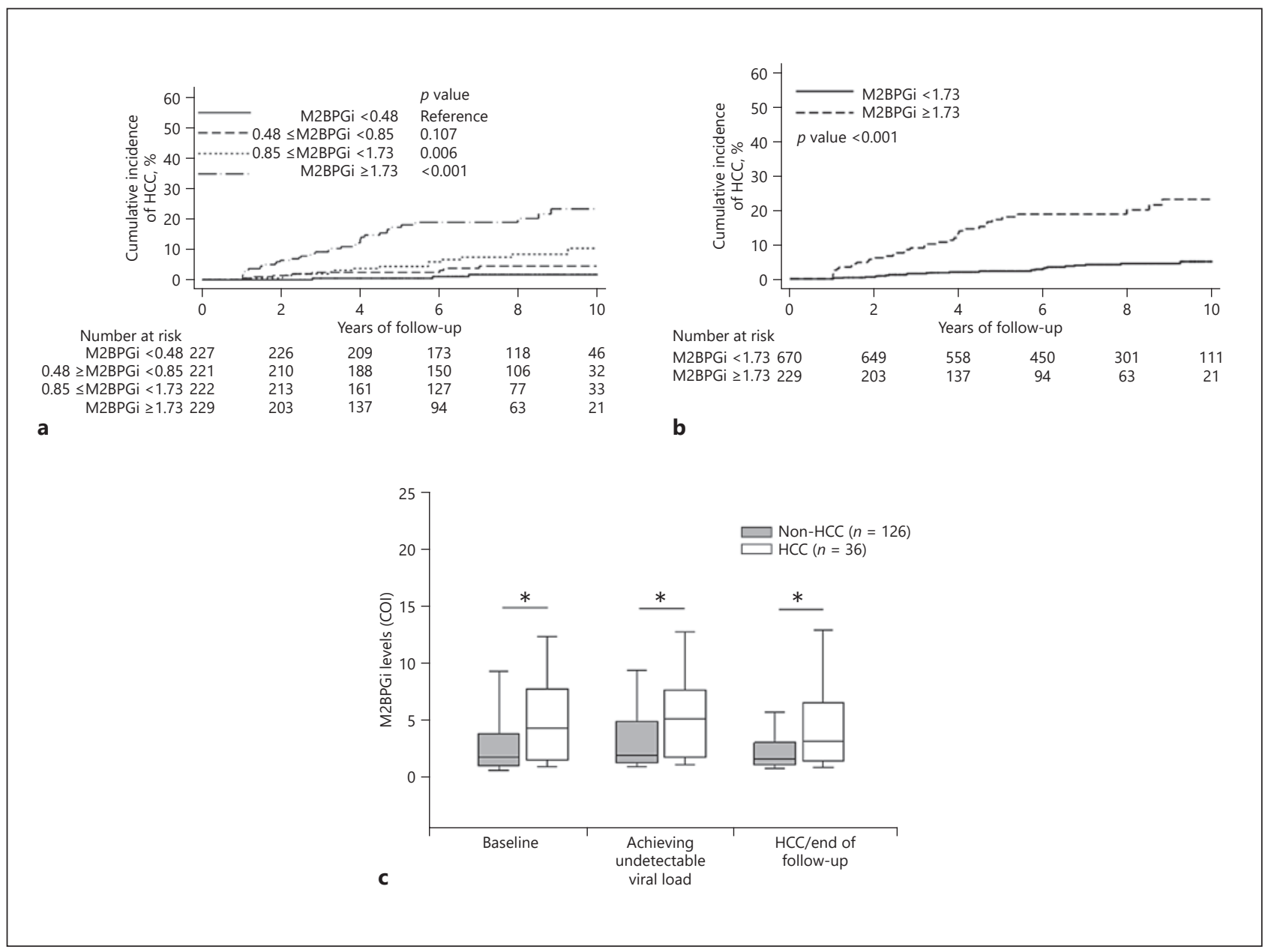

Fig. 1. In the discovery cohort of 899 chronic hepatitis B patients receiving long-term entecavir treatment, (a) patients with higher M2BPGi levels were associated with increased cumulative HCC incidence after categorizing the patients with M2BPGi quartiles. b When stratifying the patients with an M2BPGi level of 1.73 (third quartile), the high M2BPGi group was associated with an increased HCC risk when compared to the low M2BPGi group (c) When comparing the M2BPGi kinetics in patients with $(n=36)$ and without HCC $(n=$ 126) development, M2BPGi levels of the HCC group were consistently higher at different time points; * $p<$ 0.05. HCC, hepatocellular carcinoma; M2BPGi, Mac-2 binding protein glycosylation isomer.

M2BPGi $\geq 1.73$ as an HCC Risk Factor in Patients with or without Cirrhosis, Respectively

As cirrhosis is the strongest HCC risk factor, we explored whether the cutoff of 1.73 consistently stratified the HCC risks in patients with and without cirrhosis, respectively. The patients were first divided by baseline cirrhosis status, which was mostly diagnosed by abdominal sonographic findings together with clinical data. There was difference of HCC risks between the patients without and with cirrhosis, the annual incidence rates of HCC were $0.41 \%$ (95\% CI $0.27-0.62 \%$ ) and 4.62\% (95\% CI 3.42-6.26\%), respectively. After stratifying the patients by an M2BPGi level of 1.73 in both groups, high M2BPGi level, compared to the low level, was consistently associated with increased HCC risks with corresponding HRs of 4.40 (95\% CI 1.90-10.20) and 2.28 (95\% CI 1.19-4.40) in non-cirrhotic and cirrhotic patients, respectively (Fig. 2a, b, and online suppl. Table 2). Multivariable analysis consistently showed that high M2BPGi level served as an independent HCC risk factor in both groups (online suppl. Table 2). 


\section{Liver Cancer}

\begin{tabular}{l|l}
\hline Liver Cancer 2020;9:207-220 \\
\hline DOI: 10.1159/000504650 & $\begin{array}{l}\text { @ 2020 The Author(s). Published by S. Karger AG, Basel } \\
\text { www.karger.com/lic }\end{array}$ \\
\hline
\end{tabular}

Tseng et al.: M2BPGi and HBV-HCC Risks

Table 2. Univariable and multivariable analyses of factors associated with HCC development in by Cox proportional hazards regression model

\begin{tabular}{|c|c|c|c|c|c|}
\hline & $\begin{array}{l}\text { P-yrs of } \\
\text { follow-up }\end{array}$ & $\begin{array}{l}\text { HCC } \\
\text { Num }\end{array}$ & $\begin{array}{l}\text { Annual } \\
\text { incidence rate } \\
\text { (per } 100 \text { P-yrs) }\end{array}$ & $\begin{array}{l}\text { Crude HR } \\
(95 \% \mathrm{CI})\end{array}$ & $\begin{array}{l}\text { Adjusted HR (95\% } \\
\text { CI) }\end{array}$ \\
\hline \multicolumn{6}{|l|}{ Gender } \\
\hline Female & $1,476.46$ & 15 & 1.02 & 1.0 & 1.0 \\
\hline Male & $4,826.70$ & 49 & 1.02 & $1.03(0.58-1.85)$ & $1.99^{\dagger}(1.04-3.80)$ \\
\hline Age (per 1 year increased) & & & & $1.07 \S(1.05-1.09)$ & $1.06^{\S}(1.03-1.09)$ \\
\hline \multicolumn{6}{|l|}{ Serum ALT level, U/L } \\
\hline$<40$ & 856.42 & 13 & 1.52 & 1.0 & 1.0 \\
\hline$\geq 40$ & $5,446.74$ & 51 & 0.94 & $0.64(0.35-1.18)$ & $0.91(0.47-1.74)$ \\
\hline Platelet (per $10 \times 10^{9} / \mathrm{L}$ increased) & & & & $0.83^{\S}(0.79-0.87)$ & $0.99^{\dagger}(0.99-1.00)$ \\
\hline \multicolumn{6}{|l|}{ HBeAg } \\
\hline Negative & $3,556.85$ & 44 & 1.24 & 1.0 & 1.0 \\
\hline Positive & $2,746.31$ & 20 & 0.73 & $0.63(0.37-1.07)$ & $1.61(0.86-3.00)$ \\
\hline Serum HBV DNA & & & & $0.91(0.79-1.04)$ & $1.05(0.88-1.24)$ \\
\hline \multicolumn{6}{|l|}{ (Per $1 \log 10 \mathrm{IU} / \mathrm{mL}$ increased) } \\
\hline \multicolumn{6}{|l|}{ Baseline cirrhosis } \\
\hline No & $5,394.73$ & 22 & 0.41 & 1.0 & 1.0 \\
\hline Yes & 908.43 & 42 & 4.62 & $11.00^{\S}(6.50-18.59)$ & $5.10^{\S}(2.70-9.67)$ \\
\hline \multicolumn{6}{|l|}{ M2BPGi (COI) } \\
\hline$<1.73$ & $4,965.89$ & 25 & 0.50 & 1.0 & 1.0 \\
\hline$\geq 1.73$ & $1,337.27$ & 39 & 2.92 & $5.80^{\S}(3.50-9.60)$ & $2.12^{\dagger}(1.17-3.86)$ \\
\hline
\end{tabular}

${ }^{\dagger} p<0.05,{ }^{\ddagger} p<0.01,{ }^{\S} p<0.001$. P-yrs, person-years; HR, hazard ratio; ALT, alanine transaminase; HBeAg, hepatitis B e antigen; HBV, hepatitis B virus; HCC, hepatocellular carcinoma; M2BPGi, Mac-2 binding protein Glycosylation isomer; COI, cut-off index.

Sensitivity Analysis of the Patients without Liver Cirrhosis Documented by Liver Histology Data

We performed a sensitivity analysis by restricting our study population to 274 patients who received liver biopsy before entecavir treatment to determine the robustness of our findings. There were $64,78,66$, and 66 patients diagnosed as METAVIR fibrosis stages of F0/1, F2, F3, and F4, respectively. Among the non-cirrhotic patients diagnosed by histology (fibrosis stage $<$ F4), M2BPGi $\geq 1.73$, when compared to those with M2BPGi $<1.73$, was associated with a higher risk of HCC with an HR of 4.79 (95\% CI 1.38-16.55). The positive relationship persisted even when restricting the study population to those with F2 or F3 ( $p=$ 0.037). For the patients with cirrhosis diagnosed by histology, there was no significant difference in HCC risks between patients with high and low M2BPGi levels due to the small sample size.

\section{M2BPGi $\geq 1.73$ as an HCC Co-Predictor for PAGE-B Score}

The PAGE-B score is a well-validated HCC prediction model developed in Caucasian patients. We thus explored whether the PAGE-B score could stratify HCC risks in Asian CHB patients and whether M2BPGi could complement the PAGE-B score to predict HCC development.

We first correlated the PAGE-B score and HCC incidence, and showed a dose-response relationship between PAGE-B score and HCC risk ( $p$ for trend $<0.001$ ). The AUROC of PAGE-B score in predicting HCC development was 0.74 (95\% CI 0.68-0.80). Patients with M2BPGi $\geq 1.73$, when compared to $<1.73$, was consistently associated with an increased HCC risk after adjustment for PAGE-B score (HR: 4.06 with 95\% CI 2.42-6.83). 


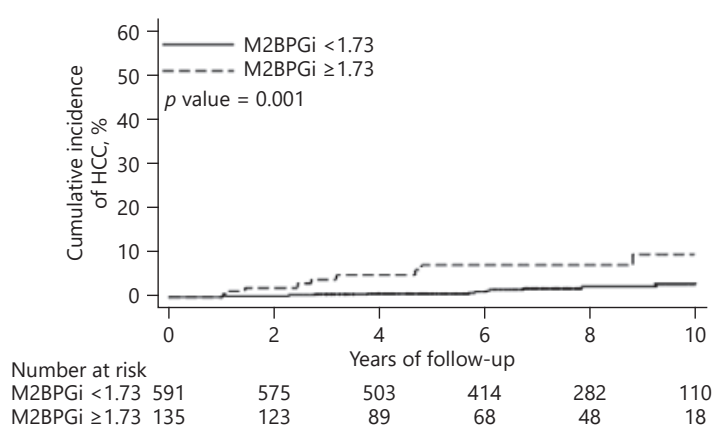

a

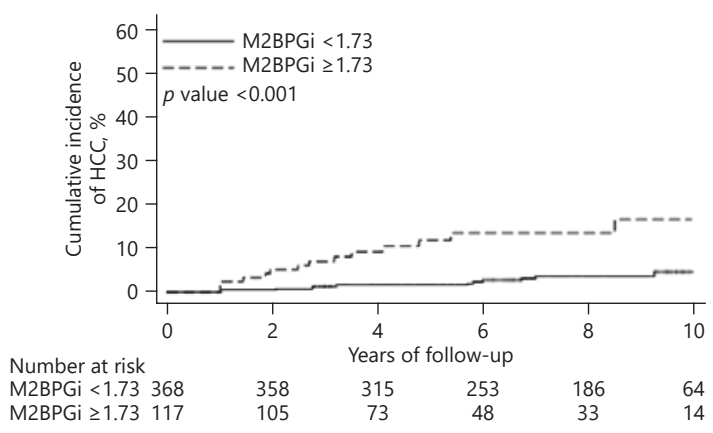

C

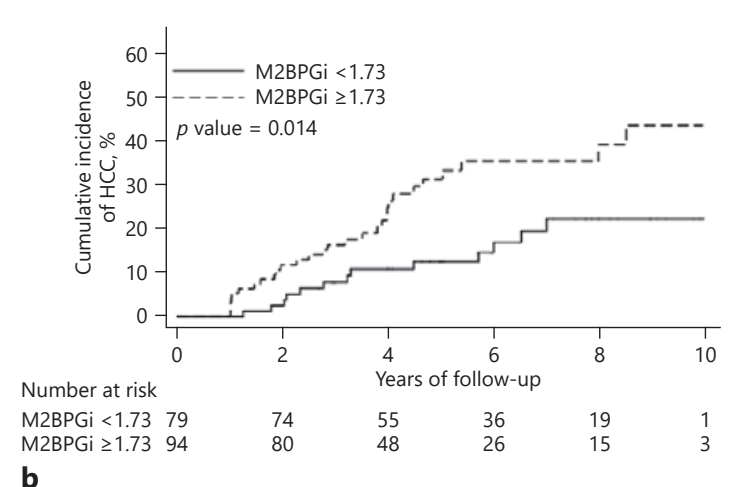

b

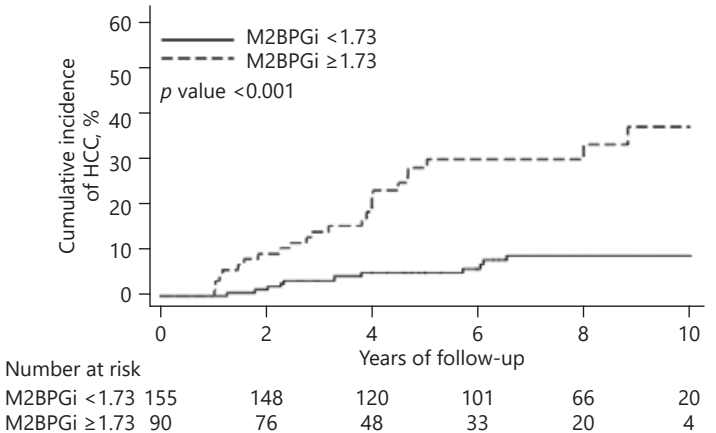

d

Fig. 2. M2BPGi level of 1.73 consistently stratified HCC risks not only in patients (a) without $(n=726)$ and (b) with $(n=173)$ cirrhosis at baseline but also in patients with (c) intermediate PAGE-B score $(n=485)$ and (d) high PAGE-B score $(n=245)$ of the discovery cohort. HCC, hepatocellular carcinoma; M2BPGi, Mac-2 binding protein glycosylation isomer.

When stratifying all the patients into 3 groups by PAGE-B model: low $(n=169, \mathrm{HCC}=2$, score $<10)$, intermediate $(n=485$, HCC $=26$, score: $10-17)$, and high $(n=245$, HCC $=36$, score $>17$ ), the corresponding annual incidence of HCC was $0.16 \%$ (95\% CI $0.04-0.64 \%$ ), $0.75 \%$ (95\% CI $0.51-1.10 \%$ ), and $2.29 \%$ (95\% CI 1.65-3.17\%) respectively.

We then explored whether the M2BPGi level was useful in stratifying HCC risks in patients with intermediate and high PAGE-B scores. The patients with M2BPGi $\geq 1.73$, compared to those with M2BPGi $<1.73$, were consistently associated with increased HCC risks in the intermediate-score group and high-score group with the corresponding HRs of 4.40 (95\% CI 1.89-10.20, Fig. 2c) and 5.13 (95\% CI 2.52-10.47, Fig. 2d) respectively. There was no role of M2BPGi in patients with low PAGE-B score as there were 2 events only.

External Validation of the M2BPGi Cut-Off Point in an Independent Cohort

The fitness of the M2BPGi cutoff was tested in an independent cohort. The baseline characteristics are summarized in Table 1 . In brief, the cohort was predominantly male $(n=282$, $73.4 \%$ ) with a median age of 46.49 years, both of which do not differ from the discovery cohort. Of note, there were patients with more severe liver fibrosis in the validation cohort as compared with the discovery cohort, which is supported by the lower platelet count 154.38 vs. $173.0010^{3} / \mu \mathrm{L}, p<0.0001$ ), more patients with serum level of M2BPGi $\geq 1.73$ (49.22 vs. 
Tseng et al.: M2BPGi and HBV-HCC Risks

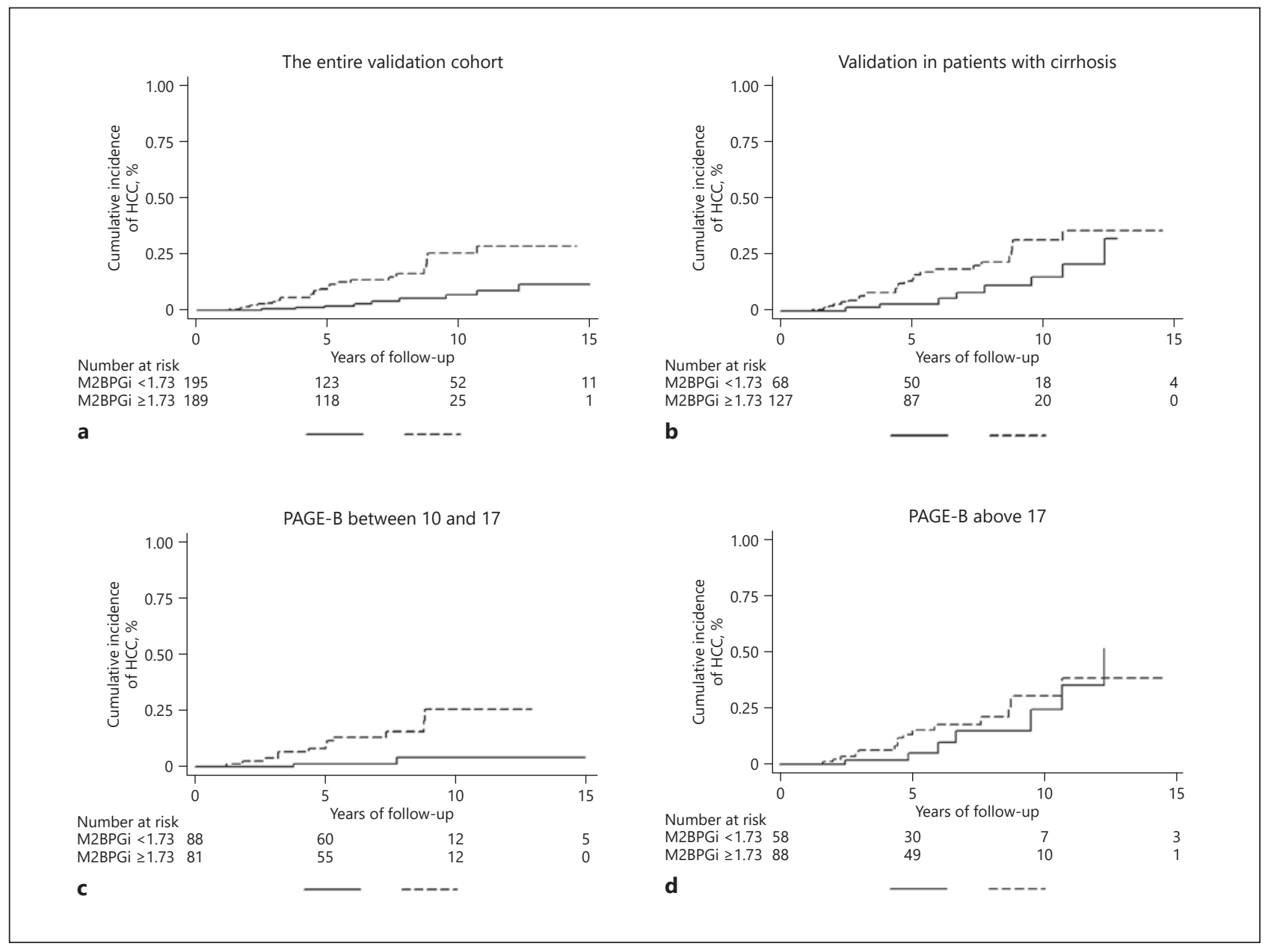

Fig. 3. In the validation cohort, M2BPGi level of 1.73 stratified HCC risks in the (a) overall patients $(n=384)$, (b) patients with liver cirrhosis $(n=195)$, (c) patients with intermediate PAGE-B $(n=169)$, but not in (d) patients with a high PAGE-B score $(n=146)$. HCC, hepatocellular carcinoma; M2BPGi, Mac-2 binding protein glycosylation isomer.

$25.47 \%, p=0.0001$ ), and more patients with liver cirrhosis (50.78 vs. $19.24 \%, p=0.0001$ ) in the validation cohort. After a mean follow-up period of $6.73 \pm 3.78$ years, HCC occurred in 36 patients in the validation cohort, almost all of whom $(n=35)$ were diagnosed with cirrhosis at baseline.

In the entire validation cohort (Fig. 3a), patients with a baseline M2BPGi level $\geq 1.73$ had a higher incidence of HCC than those with a level $<1.73(p=0.0002)$. Among patients with liver cirrhosis (Fig. 3b), the M2BPGi stratified the HCC risks with a marginal statistical significance $(p=0.05)$. Validation was not performed in patients without cirrhosis because there was only 1 event in the subgroup.

The performance of this M2BPGi cutoff was further examined in the validation cohort according to the PAGE-B score. In patients with an intermediate PAGE-B score between 10 and 17 points (Fig. 3c), M2BPGi level above 1.73 predicted a significantly higher risk of HCC $(p=0.0002)$. In those with a PAGE-B score above 17 points (Fig. $3 \mathrm{~d}$ ), however, the incidences of HCC were similarly high regardless of the baseline M2BPGi level $(p=0.4)$. The performance of M2BPGi was not examined in patients with a PAGE-B score below 10 points as no patients in this low-risk subgroup developed HCC during the follow-up. 
Tseng et al.: M2BPGi and HBV-HCC Risks

\section{Discussion}

Long-term NA treatment has been shown to diminish the risks of cirrhosis-related complications. However, the residual HCC risk remains [35]. It is an urgent need to predict the HCC risks in CHB patients receiving long-term NA treatment. Our data showed that M2BPGi was an independent predictor of HCC in CHB patients receiving long-term NA treatment. We successfully used an M2BPGi level of 1.73 to stratify the HCC risks either in the overall cohort or in the patients with and without cirrhosis, respectively. The role of M2BPGi was validated in non-cirrhotic patients with liver histology available. When stratifying the patients by PAGE-B score of 10 and 17, we found that M2BPGi levels could complement the PAGE-B model in predicting HCC development, especially in those with intermediate scores. Finally, we extrapolated our findings and examined the performance of the M2BPGi cutoff in an independent cohort. In this validation cohort with more cirrhotic patients, a baseline M2BPGi level above 1.73 was consistently predictive of a higher HCC risk, particularly those with intermediate PAGE-B score. Taking all these lines of evidence together, M2BPGi is a novel biomarker to predict HCC risks in CHB patients receiving long-term NA and the cutoff of 1.73 is useful in stratifying their HCC risks.

The key to optimizing the clinical management of CHB patients receiving long-term NA treatment is to precisely define their HCC risks. The most important finding of this study is that for the first time, we showed a single M2BPGi cutoff supplements current HCC prediction models in stratifying HCC risks, which is summarized in online supplementary Table 2. For example, in patients without liver cirrhosis of discovery cohort, $88 \%$ of them had low M2BPGi levels with an annual HCC incidence rate of $0.26 \%$ in contrast to the annual incidence of $1.1 \%$ in those with high M2BPGi levels. In patients with intermediate PAGE-B score, their annual incidence rate dropped from 1.96 to $0.43 \%$ in discovery cohort after stratifying them with M2BPGi levels of 1.73. The similar effect of risk stratification was validated in the independent validation cohort with annual incidence rates of 2.28 and $0.30 \%$ respectively. Although the HCC risks of both low M2BPGi groups were still higher than $0.2 \%$ per year, which is the recommended threshold for not initiating HCC surveillance [24], adding this new criterion definitely lowers the HCC risks in different patient populations. In other words, a low M2BPGi level paves the way for identifying the patients with a minimal HCC risk, who may not need HCC surveillance.

It has been shown that M2BPGi level is associated with development of HBV-related HCC in different treatment cohort [19-23]. The M2BPGi cutoff ranged from 0.34 to 1.80 . To the best of our knowledge, our study has the most patients receiving antiviral treatment and is the first one with an independent validation cohort to support our finding and cutoff. In addition, we are also the first group to show that M2BPGi level of 1.73 complements PAGE-B score in predicting HCC development, which can be applied in clinical practice immediately. To be noted, we chose the third M2BPGi quartile of 1.73 as our cutoff, which is very close to the cutoff of 1.80 proposed by the Korean group to stratify HCC risks [20]. In addition, M2BPGi level of 1.79 has been proposed as a useful cutoff to differentiate the patients with fibrosis stage of F3 or more [34]. Taking all these lines of evidence together, we believe M2BPGi level of 1.73 is a useful biomarker in predicting risks of HBV-related HCC.

Another interesting finding is that we used a single M2BPGi cutoff to stratify HCC risks in CHB patients with different liver fibrosis severity. A similar finding has also been shown in a Japanese cohort of chronic hepatitis C patients [17]. Both data imply that M2BPGi may serve not only as a marker of liver fibrosis but also an early marker associated with carcinogenesis. Recent data suggest that M2BPGi, which is mainly secreted by hepatic stellate cells, not only serves as a messenger between hepatic stellate cell and Kupffer cell during fibrogenesis but may also exert biological activity on other Mac-2-positive non-parenchymal cells in liver [16]. 


\section{Liver

The interaction between M2BPGi and M2 protein may alter the liver microenvironment, which plays a significant role in liver carcinogenesis [16]. However, we did not see a surge of M2BPGi level before HCC development (Fig. 1c), thus it might not serve as a late marker for HCC diagnosis.

Although viral factors have been shown to be associated with HCC development in treatment-naïve patients, it remains unclear how the viral factors affect HCC development in on-treatment patients. In this study, we first investigated the relationship between pretreatment viral factors and HCC risk. Our data showed there was no association between baseline HBV DNA levels and HCC risks in 899 patients (Table 2). In addition, we also analyzed the baseline HBsAg levels and HCC risks in 487 patients with available data. We found that HBsAg levels were not associated with HCC development, either (HR for per $1 \log _{10} \mathrm{IU} / \mathrm{mL}$ increase of HBsAg: 0.77 [95\% CI 0.56-1.05]). Both findings were consistent with previous reports $[6,7,36,37]$. Hepatitis core-related antigen is another novel biomarker. Accumulating data suggest that its level is highly associated with HCC development in treatmentnaïve patients [38, 39], while limited reports have shown its role in predicting HCC risk among on-treatment patients [37,40]. More studies are thus needed.

Another important viral factor is whether earlier achievement of undetectable viral load is associated with a lower risk of HCC development. As all of the patients in this study received entecavir treatment, a potent NA, 73.6 and $88.8 \%$ of the patients with available data achieved undetectable viral load at 6 months and 12 months of entecavir therapy, respectively. Univariable and multivariable analyses showed that achieving undetectable viral load at the early phase of entecavir therapy was not associated with HCC development, which has been presented in a previous study by Chen et al. [6].

There are a few limitations in this study. First, liver stiffness measurement was not used widely to diagnose liver cirrhosis when enrolling our patients. However, our main finding was the independent role of M2BPGi from the PAGE-B-score, which does not include cirrhosis as a variable [7]. Thus, such a limitation did not affect the complementary role of M2BPGi. Second, our findings were mostly confirmed in the validation cohort except in the patients with high PAGE-B group patients. Carcinogenesis is more complex in these high-risk patients and other factors, such as accumulation of host genome mutations and viral genome integration, could be more important than fibrosis stage. We believe that is why M2BPGi does not predict HCC well in these high-risk patients. Third, all of our patients, including validation cohort, were Asian patients with either genotype B or C infection, more data from Caucasian patients are needed to validate the global usefulness of M2BPGi level. Fourth, we excluded the patients with HCC development within the first year of follow-up, which precludes the possibility of investigating whether M2BPGi could predict HCC development during the early phase after NA initiation. Last, as we did not enroll the patients without antiviral therapy, we need more data to validate the proposed M2BPGi cutoff in the treatmentnaïve patients.

In summary, among CHB patients receiving long-term antiviral treatment, a higher baseline M2BPGi level is associated with an increased HCC risk. An M2BPGi level of 1.73 is useful in stratifying HCC risks, especially in those with intermediate PAGE-B scores, which has been confirmed in an independent cohort.

\section{Acknowledgments}

We thank Sysmex company for providing the M2BPGi kits for M2BPGi measurement. We also thank Mr. Cheng-Hsueh Tsai from department of internal medicine of NTUH for determining all the viral biomarkers and M2BPGi levels. Finally, we thank the Cancer Registry, Cancer Administration and Coordination Center, NTUH for providing cancer registration data to confirm our diagnoses. 
Tseng et al.: M2BPGi and HBV-HCC Risks

\section{Statement of Ethics}

Our research complies with the guidelines for human studies and has been conducted ethically in accordance with the World Medical Association Declaration of Helsinki. All the enrolled patients in both cohorts gave written informed consent as approved by the research Ethical Committees of each hospital.

\section{Disclosure Statement}

T.-C.T. has served on speaker's bureaus for Abbvie, Bristol-Myers Squibb, and Gilead Sciences. Y.-C.H. has received lecture fees from Abbvie, Bristol-Myers Squibb, Gilead Sciences, and Novartis, and served as an advisory committee member for Gilead Sciences. M.H.N. Research support: Janssen, Gilead, Pfizer; Advisory board/consulting: Novartis, Janssen, Gilead, Spring Bank, Bayer, Eisai, Exact Sciences, Laboratory of Advanced Medicine. M.-L.Y. Research support: Abbvie, BMS, Gilead, Torpedo and Merck; consultant for Abbvie, Abbott, Ascletis, BMS, Gilead, Merck, and Pharmaessential; speaker for Abbvie, Abbott, Ascletis, BMS, Gilead, and Merck. J.-H.K. has served as a consultant for Abbvie, Gilead Sciences, Merck Sharp and Dohme, and Roche and on speaker's bureaus for Abbvie, Bristol-Myers Squibb, Gilead Sciences, Merck Sharp and Dohme. Others claimed no conflicts of interest.

\section{Funding Sources}

This work was supported by the grants from National Taiwan University Hospital (106-N3626, 107-S3816, and 107-N4041), the Ministry of Science and Technology, Executive Yuan, Taiwan (MOST 105-2314-B-303-008 and MOST 106-2314-B-002-136), and the National Health Research Institutes (NHRIEX108-10807BC).

\section{Author Contributions}

T.C.-T., C.-Y.P., Y.-C.H., and J.-H.K: study concept and design. T.-C.T., C.-Y.P., Y.-C.H., M.-L.Y., C.-J.L., C.-H.L., Y.T., H.-C.Y., T.-H.S., H.-C.L., C.-H.L., P.-J.C., D.-S.C., C.-C.W., and J.-H.K.: acquisition of data. T.-C.T., C.-Y.P., Y.-C.H., M.H.N., and J.-H.K.: analysis and interpretation of data. T.-C.T.: drafting of the manuscript. C.-Y.P., and J.-H.K.: critical review of the manuscript for important intellectual content. T.-C.T., C.-Y.P., and Y.-C.H.: statistical analysis. T.-C.T., C.-J.L., and J.-H.K.: obtained funding. T.-C.T. and Y.T.: technical or material support. C.-Y.P. and J.-H.K.: study supervision.

\section{References}

1 Kao JH. Hepatitis B: from control to cure. J Formos Med Assoc. 2018 Oct;117(10):868-70.

2 Wong GL, Chan HL, Mak CW, Lee SK, Ip ZM, Lam AT, et al. Entecavir treatment reduces hepatic events and deaths in chronic hepatitis B patients with liver cirrhosis. Hepatology. 2013 Nov;58(5):1537-47.

3 Su TH, Hu TH, Chen CY, Huang YH, Chuang WL, Lin CC, et al.; C-TEAM study group and the Taiwan Liver Diseases Consortium. Four-year entecavir therapy reduces hepatocellular carcinoma, cirrhotic events and mortality in chronic hepatitis B patients. Liver Int. 2016 Dec;36(12):1755-64.

4 Wong VW, Janssen HL. Can we use HCC risk scores to individualize surveillance in chronic hepatitis B infection? J Hepatol. 2015 Sep;63(3):722-32.

5 Tseng TC, Liu CJ, Su TH, Yang WT, Chen CL, Yang HC, et al. Fibrosis-4 Index Helps Identify HBV Carriers With the Lowest Risk of Hepatocellular Carcinoma. Am J Gastroenterol. 2017 Oct;112(10):1564-74.

6 Chen CH, Lee CM, Lai HC, Hu TH, Su WP, Lu SN, et al. Prediction model of hepatocellular carcinoma risk in Asian patients with chronic hepatitis B treated with entecavir. Oncotarget. 2017 Sep;8(54):92431-41.

7 Papatheodoridis G, Dalekos G, Sypsa V, Yurdaydin C, Buti M, Goulis J, et al. PAGE-B predicts the risk of developing hepatocellular carcinoma in Caucasians with chronic hepatitis B on 5-year antiviral therapy. J Hepatol. 2016 Apr;64(4):800-6.

8 Wong GL, Chan HL, Wong CK, Leung C, Chan CY, Ho PP, et al. Liver stiffness-based optimization of hepatocellular carcinoma risk score in patients with chronic hepatitis B. J Hepatol. 2014 Feb;60(2):339-45.

9 Yuen MF, Tanaka Y, Fong DY, Fung J, Wong DK, Yuen JC, et al. Independent risk factors and predictive score for the development of hepatocellular carcinoma in chronic hepatitis B. J Hepatol. 2009 Jan;50(1):80-8. 
Tseng et al.: M2BPGi and HBV-HCC Risks

10 Wong VW, Chan SL, Mo F, Chan TC, Loong HH, Wong GL, et al. Clinical scoring system to predict hepatocellular carcinoma in chronic hepatitis B carriers. J Clin Oncol. 2010 Apr;28(10):1660-5.

11 Arends P, Sonneveld MJ, Zoutendijk R, Carey I, Brown A, Fasano M, et al.; VIRGIL Surveillance Study Group. Entecavir treatment does not eliminate the risk of hepatocellular carcinoma in chronic hepatitis B: limited role for risk scores in Caucasians. Gut. 2015 Aug;64(8):1289-95.

12 Jung KS, Kim SU, Song K, Park JY, Kim DY, Ahn SH, et al. Validation of hepatitis B virus-related hepatocellular carcinoma prediction models in the era of antiviral therapy. Hepatology. 2015 Dec;62(6):1757-66.

13 Wong GL, Chan HL, Chan HY, Tse PC, Tse YK, Mak CW, et al. Accuracy of risk scores for patients with chronic hepatitis B receiving entecavir treatment. Gastroenterology. 2013 May;144(5):933-44.

14 Kim MN, Hwang SG, Rim KS, Kim BK, Park JY, Kim DY, et al. Validation of PAGE-B model in Asian chronic hepatitis B patients receiving entecavir or tenofovir. Liver Int. 2017 Dec;37(12):1788-95.

15 European Association for Study of LiverAsociacion Latinoamericana para el Estudio del Higado. EASL-ALEH Clinical Practice Guidelines: non-invasive tests for evaluation of liver disease severity and prognosis. J Hepatol. 2015 Jul;63(1):237-64.

16 Shirabe K, Bekki Y, Gantumur D, Araki K, Ishii N, Kuno A, et al. Mac-2 binding protein glycan isomer (M2BPGi) is a new serum biomarker for assessing liver fibrosis: more than a biomarker of liver fibrosis. J Gastroenterol. 2018 Jul;53(7):819-26.

17 Yamasaki K, Tateyama M, Abiru S, Komori A, Nagaoka S, Saeki A, et al. Elevated serum levels of Wisteria floribunda agglutinin-positive human Mac-2 binding protein predict the development of hepatocellular carcinoma in hepatitis C patients. Hepatology. 2014 Nov;60(5):1563-70.

18 Hsu YC, Jun T, Huang YT, Yeh ML, Lee CL, Ogawa S, et al. Serum M2BPGi level and risk of hepatocellular carcinoma after oral anti-viral therapy in patients with chronic hepatitis B. Aliment Pharmacol Ther. 2018 Nov; 48(10):1128-37.

19 Kawaguchi K, Honda M, Ohta H, Terashima T, Shimakami T, Arai K, et al. Serum Wisteria floribunda agglutininpositive Mac-2 binding protein predicts hepatocellular carcinoma incidence and recurrence in nucleos(t)ide analogue therapy for chronic hepatitis B. J Gastroenterol. 2018 Jun;53(6):740-51.

20 Kim SU, Heo JY, Kim BK, Park JY, Kim DY, Han KH, et al. Wisteria floribunda agglutinin-positive human Mac-2 binding protein predicts the risk of HBV-related liver cancer development. Liver Int. 2017 Jun; 37(6):879-87.

21 Shinkai N, Nojima M, Iio E, Matsunami K, Toyoda H, Murakami S, et al. High levels of serum Mac-2-binding protein glycosylation isomer (M2BPGi) predict the development of hepatocellular carcinoma in hepatitis B patients treated with nucleot(s)ide analogues. J Gastroenterol. 2018 Jul;53(7):883-9.

22 Cheung KS, Seto WK, Wong DK, Mak LY, Lai CL, Yuen MF. Wisteria floribunda agglutinin-positive human Mac-2 binding protein predicts liver cancer development in chronic hepatitis B patients under antiviral treatment. Oncotarget. 2017 Jul;8(29):47507-17.

23 Mak LY, Ko M, To E, Wong DK, Ma JH, Hui TL, et al. Serum Mac-2-binding protein glycosylation isomer and risk of hepatocellular carcinoma in entecavir-treated chronic hepatitis B patients. J Gastroenterol Hepatol. 2019 Oct;34(10):1817-23.

24 Terrault NA, Lok AS, McMahon BJ, Chang KM, Hwang JP, Jonas MM, et al. Update on prevention, diagnosis, and treatment of chronic hepatitis B: AASLD 2018 hepatitis B guidance. Hepatology. 2018 Apr;67(4):1560-99.

25 Lampertico P, Agarwal K, Berg T, Buti M, Janssen HL, Papatheodoridis G, et al.; European Association for the Study of the Liver. EASL 2017 Clinical Practice Guidelines on the management of hepatitis B virus infection. J Hepatol. 2017;67(2):370-98.

26 Tseng TC, Liu CJ, Yang HC, Chen CL, Yang WT, Tsai CS, et al. Higher proportion of viral basal core promoter mutant increases the risk of liver cirrhosis in hepatitis B carriers. Gut. 2015 Feb;64(2):292-302.

27 Tseng TC, Liu CJ, Su TH, Yang WT, Chen CL, Yang HC, et al. Fibrosis-4 index predicts cirrhosis risk and liverrelated mortality in 2075 patients with chronic HBV infection. Aliment Pharmacol Ther. 2018 Jun; 47(11): 1480-9.

28 Bruix J, Sherman M; American Association for the Study of Liver Diseases. Management of hepatocellular carcinoma: an update. Hepatology. 2011 Mar;53(3):1020-2.

29 Liu J, Hu HH, Lee MH, Korenaga M, Jen CL, Batrla-Utermann R, et al. Serum Levels of M2BPGi as Short-Term Predictors of Hepatocellular Carcinoma in Untreated Chronic Hepatitis B Patients. Sci Rep. 2017 Oct; 7(1): 14352.

30 Heo JY, Kim SU, Kim BK, Park JY, Kim DY, Ahn SH, et al. Use of Wisteria Floribunda Agglutinin-Positive Human Mac-2 Binding Protein in Assessing Risk of Hepatocellular Carcinoma Due to Hepatitis B Virus. Medicine (Baltimore). 2016 Apr;95(14):e3328.

31 Ichikawa Y, Joshita S, Umemura T, Shobugawa Y, Usami Y, Shibata S, et al. Serum Wisteria floribunda agglutinin-positive human Mac-2 binding protein may predict liver fibrosis and progression to hepatocellular carcinoma in patients with chronic hepatitis B virus infection. Hepatol Res. 2017 Feb;47(2):226-33.

32 Mak LY, To WP, Wong DK, Fung J, Liu F, Seto WK, et al. Serum Mac-2 binding protein glycosylation isomer level predicts hepatocellular carcinoma development in E-negative chronic hepatitis B patients. World J Gastroenterol. 2019 Mar;25(11):1398-408.

33 Toyoda H, Kumada T, Tada T, Kaneoka Y, Maeda A, Korenaga M, et al. Serum WFA+ -M2BP levels as a prognostic factor in patients with early hepatocellular carcinoma undergoing curative resection. Liver Int. 2016 Feb;36(2):293-301. 
34 Nishikawa H, Enomoto H, Iwata Y, Kishino K, Shimono Y, Hasegawa K, et al. Serum Wisteria floribunda agglutinin-positive Mac-2-binding protein for patients with chronic hepatitis B and C: a comparative study. J Viral Hepat. 2016 Dec;23(12):977-84.

35 Tseng TC, Kao JH. Elimination of Hepatitis B: Is It a Mission Possible? BMC Med. 2017 Mar;15(1):53.

36 Brichler S, Nahon P, Zoulim F, Layese R, Bourcier V, Audureau E, et al.; ANRS C012 CirVir study group. Nonvirological factors are drivers of hepatocellular carcinoma in virosuppressed hepatitis B cirrhosis: results of ANRS C012 CirVir cohort. J Viral Hepat. 2019 Mar;26(3):384-96.

37 Cheung KS, Seto WK, Wong DK, Lai CL, Yuen MF. Relationship between HBsAg, HBcrAg and hepatocellular carcinoma in patients with undetectable HBV DNA under nucleos(t)ide therapy. J Viral Hepat. 2017 Aug;24(8): 654-61.

38 Tada T, Kumada T, Toyoda H, Kiriyama S, Tanikawa M, Hisanaga Y, et al. HBcrAg predicts hepatocellular carcinoma development: an analysis using time-dependent receiver operating characteristics. J Hepatol. 2016 Jul;65(1):48-56.

39 Tseng TC, Liu CJ, Hsu CY, Hong CM, Su TH, Yang WT, et al. High Level of Hepatitis B Core-Related Antigen Associated With Increased Risk of Hepatocellular Carcinoma in Patients With Chronic HBV Infection of Intermediate Viral Load. Gastroenterology. 2019 Dec;157(6):1518-29.e3.

40 Hosaka T, Suzuki F, Kobayashi M, Fujiyama S, Kawamura Y, Sezaki H, et al. Impact of hepatitis B core-related antigen on the incidence of hepatocellular carcinoma in patients treated with nucleos(t)ide analogues. Aliment Pharmacol Ther. 2019 Feb;49(4):457-71. 